\title{
Belphégor
}

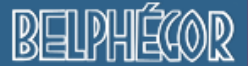

Littérature populaire et culture médiatique

19-2 | 2021

La civilisation du magazine

\section{Jean Marigny. Les enfants de l'imaginaire}

\section{Benoît Peuch}

\section{(2) OpenEdition}

Journals

Édition électronique

URL : https://journals.openedition.org/belphegor/4319

DOI : 10.4000/belphegor.4319

ISSN : 1499-7185

Éditeur

LPCM

\section{Référence électronique}

Benoît Peuch, « Jean Marigny. Les enfants de l'imaginaire », Belphégor [En ligne], 19-2 | 2021, mis en

ligne le 04 janvier 2022, consulté le 20 septembre 2022. URL : http://journals.openedition.org/ belphegor/4319; DOI : https://doi.org/10.4000/belphegor.4319

Ce document a été généré automatiquement le 20 septembre 2022.

\section{(c) (i) (9)}

Creative Commons - Attribution - Pas d'Utilisation Commerciale - Pas de Modification 4.0 International - CC BY-NC-ND 4.0

https://creativecommons.org/licenses/by-nc-nd/4.0/ 


\title{
Jean Marigny. Les enfants de l'imaginaire
}

\author{
Benoît Peuch
}

\section{RÉFÉRENCE}

Jean Marigny, Les enfants de l'imaginaire, Terre de brume, 2021, p. 267

Dans la littérature, la " découverte de l'enfant » qu'Ariès ${ }^{1}$ situait au XVIIIe siècle se fait avec un peu de retard : il faut attendre le XIX ${ }^{\mathrm{e}}$ siècle pour qu'il devienne un personnage littéraire de premier plan. Dans des romans comme Sans famille ${ }^{2}$ où Oliver Twist ${ }^{3}$, les écrivains représentent l'enfant comme un être fondamentalement bon, victime du milieu social dans lequel il vit. Ces œuvres, qui cherchent d'abord à susciter la compassion du lecteur par leur tonalité mélodramatique, se finissent généralement bien - exprimant avec optimisme la possibilité d'une réconciliation entre l'enfant et la société. À partir de la deuxième moitié du $\mathrm{XX}^{\mathrm{e}}$ siècle, une nouvelle manière de raconter l'enfance fait son apparition. Certaines œuvres littéraires, relevant généralement de la littérature " de genre ${ }^{4}$, se mettent à les dépeindre de façons hors-normes, aberrantes et, surtout, profondément dérangeantes. Certaines d'entre elles mettent en scène des enfants criminels contredisant l'habituelle représentation angélique de l'enfance. D'autres racontent l'histoire d'enfants confrontés à une violence tellement extrême qu'elle interdit toute possibilité de réconciliation future avec la société. À partir de ces œuvres, Jean Marigny forge un concept original : celui d'enfance insolite. Dans le livre, ce concept est avant tout défini de façon extensive, à partir d'une constellation de sept représentations : l'enfant victime, l'enfant criminel, l'enfant fantôme, l'enfant vampire, l'enfant démon et l'enfant superhéros. Intensivement, on pourrait définir cette enfance insolite comme un ensemble de représentations littéraires aberrantes de l'enfant produisant un effet de gêne intense sur son lecteur. Pour Marigny, s'il y a gêne, c'est parce que l'exagération littéraire force la confrontation brutale avec une dimension inacceptable de la réalité sociale - une dimension que nous avons tendance à refouler quand l'actualité nous y expose. Par-là, étudier l'enfance insolite, c'est examiner une 
forme de matérialité littéraire qui force son lecteur à un certain niveau de lucidité sociale en le poussant aux limites de ce que sa sensibilité morale peut supporter.

2 La première forme d'enfance insolite explorée par Marigny est celle des enfants victimes. Comme nous l'avons déjà évoqué, la littérature du XIX siècle représentait déjà l'enfant comme un être victime du monde des adultes. À partir de la seconde moitié du $\mathrm{XX}^{\mathrm{e}}$ siècle, par exemple dans Une fille comme les autres de Jack Ketchum ${ }^{5}$, la victimisation de l'enfant se matérialise dans des scènes d'une violence insoutenable ayant des conséquences irréversibles. Ici, la compassion et l'espoir de réconciliation laissent la place à l'effroi et au constat cynique de l'indifférence des pouvoirs publics face à la maltraitance de l'enfant (p. 19).

En négatif de la première catégorie, viennent ensuite les enfants pervers et criminels. Dans la littérature du XIXe siècle, les petits délits des enfants sont généralement renvoyés à la mauvaise influence d'un milieu social qui néglige leurs éducations. Au XXe siècle, dans des romans comme Graine de potence $e^{6}$ de William March ou Il faut qu'on parle de Kevin ${ }^{7}$ de Lionel Shriver, l'enfant criminel est moins un enfant négligé qu'un enfant qui résiste à l'éducation qu'on lui donne. Pour Margny, ce type de récit nous renvoie à des problématiques que la criminologie phrénologique du XVIIIe siècle posait déjà : celle de la naturalité du mal (p. 67).

Suivent les enfants sauvages ou livrés à eux-mêmes. Dans cette catégorie, il n'est pas question d'enfants sauvages comme pouvait l'être Victor de l'Aveyron, mais plutôt de récits mettant en scène un groupe d'enfants se retrouvant brusquement isolés du monde des adultes. Sur cette thématique, Marigny met en avant l'existence de deux catégories de récits. Dans la première, qui survit au XIX ${ }^{\mathrm{e}}$ siècle et trouve son modèle emblématique dans Deux ans de vacances de Jules Verne ${ }^{8}$, les enfants deviennent les fondateurs d'une société harmonieuse où les rivalités finissent toujours par se régler au profit de l'intérêt commun. La deuxième catégorie, illustrée par Quinzinzinzili de Régis Messac $^{9}$ ou Sa Majesté les Mouches de William Golding ${ }^{10}$, met en scène l'échec du contrat social : la société enfantine tournant au conflit généralisé ou à la dictature. De cette division, Marigny montre comment les auteurs utilisent la robinsonnade comme une expérience de pensée littéraire mettant à l'épreuve nos attentes vis-à-vis de l'éducation. Ainsi, dans la version optimiste de Jules Verne, le récit corrobore l'idéal républicain d'une pacification de l'homme par l'éducation. Dans la version pessimiste, qui commence à voir le jour après la Première Guerre, le récit tend vers le constat désabusé de l'inéducabilité fondamentale de l'homme.

5 Les quatre catégories suivantes relèvent plus spécifiquement de la littérature fantastique. Viennent d'abord les fantômes d'enfant, une représentation qui apparaît au XIXe siècle dans la littérature victorienne. Dans ces œuvres, l'enfant fantôme est généralement un enfant victime qui revient d'entre les morts pour réparer pacifiquement un tort qu'il a subi. À partir du $\mathrm{XX}^{\mathrm{e}}$ siècle, la recherche de réconciliation laisse la place au désir de vengeance : l'enfant fantôme n'est plus un enfant triste, mais un enfant en colère qui se retourne contre la société qui l'a détruit.

Les enfants vampires n'existent pas dans la littérature avant le $\mathrm{XX}^{\mathrm{e}}$ siècle. À partir de l'analyse d'Entretien avec un vampire d'Anne Rice ${ }^{11}$ et de Laisse-moi entrer de John Ajvide Lindqvist $^{12}$, Marigny montre que le personnage de l'enfant vampire est souvent représenté comme un individu qui devient adulte en conservant éternellement un corps d'enfant qui lui interdit l'accès à la sexualité : la violence de l'enfant vampire est 
ainsi la manifestation d'une frustration qui, suggère Marigny, symbolise celle de l'adolescent face à des parents s'opposant à ce qu'il ait une vie sexuelle.

Dans le cas des enfants démoniaques, Marigny dégage deux types récits: d'un côté les histoires d'enfant possédés, comme dans L'exorciste de William Blatty ${ }^{13}$, de l'autre celle d'enfants engendrés par le diable, comme dans Un bébé pour Rosemary d'Ira Levin ${ }^{14}$. Selon Marginy, dans le premier cas, l'enfant est représenté de façon ambigüe, comme un être qui, dépossédé de lui-même, est à la fois agent et patient d'un mal radical. Dans le second, cette ambiguïté disparaît : l'enfant démon est un être absolument mauvais, un avatar de l'Antéchrist conduisant l'humanité à sa perte (p. 201).

Le livre se ferme sur le cas des enfants aux pouvoirs extraordinaires. Dans ces récits, des enfants particulièrement vulnérables - soit parce qu'ils sont handicapés (comme dans L'Enfant de cristal de Theodore Roszak ${ }^{15}$ ), soit parce que ce sont des enfants victimes (comme dans Shining de Stephen King ${ }^{16}$ ) - qui se mettent à développer un pouvoir particulier leur permettant de se défendre contre ce qui les menace. Mais, l'enfant risquant à tout moment d'être dépassé par la puissance de ses pouvoirs, devient dangereux pour lui-même et pour la société.

C'est après cette traversée thématique des représentations de l'enfance dans la littérature de genre que Marigny en vient, dans la conclusion, à faire émerger le concept d'« enfance insolite » que nous avons esquissé au début de ce compte rendu. De façon surprenante, cette conclusion si importante prend la forme fugace d'une synthèse de trois pages qui, tout en réussissant parfaitement à faire saisir les enjeux généraux de cette étude, ne peuvent que laisser le lecteur sur sa faim. Car si Marigny établit bien le fait littéraire de l'enfance insolite, on referme le livre en ayant le sentiment qu'il reste encore beaucoup de choses à en dire et qu'une théorie de l'enfance insolite reste encore à faire. Mais peut-être voulons-nous aller trop vite. La quatrième de couverture nous indique que ce livre est le "premier » ouvrage de l'auteur sur le sujet, nous laissant espérer la publication future d'un deuxième volume. En attendant, il faut convenir que Marigny a su éveiller notre curiosité en montrant comment la littérature de genre nous conduit à réfléchir sur les failles de notre société en partant des angoisses que nous éprouvons face à des œuvres qui représentent l'enfance de façon insolite.

\section{NOTES}

1. Philippe Ariès, L'enfant et la vie familiale sous l'Ancien régime, Plon, 1960.

2. Hector Malot, Sans famille, 1878.

3. Charles Dickens, Oliver Twist, 1837.

4. Par cette expression, on englobe la science-fiction, le fantastique, le roman policier et le roman noir.

5. Jack Ketchum, Une fille comme les autres (The Girl Next Door, 1989), Paris, Bragelonne, 2007. 
6. William March, Graine de potence (The Bad Seed, 1956), Paris, Gallimard, coll. "Série Noire », 1965.

7. Lionel Shriver, Il faut qu'on parle de Kevin (We Need to Talk about Kevin, 2003), Paris, J'ai Lu, 2006.

8. Jules Vernes, Deux ans de vacances, 1888.

9. Régis Messac, Quinzinzinzili (1935), Talence, édition de l'Arbre vengeur, 2011.

10. William Golding, Sa Majesté les Mouches (Lord of the Flies, 1954), Paris, Gallimard, 1956.

11. Anne Rice, Entretien avec un vampire (Interview with the Vampire, 1976), Paris, édition Jean-Claude Lattès, 1978.

12. John Ajvide Lindqvist, Laisse-moi entrer (2004), Paris, Bragelonne-Milady, 2011.

13. William Peter Blatty, L'exorciste (The Exorcist, 1971), Paris, Robert Laffont, 1971.

14. Ira Levin, Un bébé pour Rosemary (Rosemary's Baby, 1967), Paris, Robert Laffont, 1968.

15. Theodore Roszak, L'Enfant de cristal (The Crystal Child, 2007) Paris, Le Cherche Midi, 2008.

16. Stephen King, Shining (The Shining, 1977), Paris, J'ai Lu, 1981.

INDEX

Mots-clés : enfants, horreur, imaginaire

\section{AUTEUR}

BENOÎT PEUCH

Doctorant en philosophie et sciences sociales, EHESS (LIER-FYT) 